Cochrane Corner: Text messaging to improve adherence to drugs for secondary prevention of cardiovascular disease

Alma J Adler ${ }^{1}$, JP Casas ${ }^{2}$, Nicole Martin², Caroline Free ${ }^{1}$, Pablo Perel ${ }^{1}$.

${ }^{1}$ London School of Hygiene \& Tropical Medicine

${ }^{2}$ Institute of health informatics, University College London

\title{
Background.
}

Despite numerous readily available low cost and effective medications for secondary prevention of cardiovascular disease, it has been shown that a substantial proportion of cardiovascular events in people with established atherosclerotic cardiovascular disease are due to poor adherence ${ }^{1}$. Worldwide at least 100 million people are thought to have prevalent heart disease, resulting in at least 13 million deaths each year. The majority of these deaths occur in low and middle income countries $^{2}$. Given the complexity of the problem and the variety of settings it occurs in, finding a feasible and scalable intervention to increase adherence poses challenges. Globally there are over 7.5 billion mobile phone subscriptions, and even in low and middle income countries, the penetrance rate is over $90 \%$. Some mobile short messages services (SMS) have been shown to improve medication adherence for chronic conditions such as HIV3. SMS could be a potentially costeffective and scalable intervention, and do not require that the intended audience need to search for information as it is delivered to them, as opposed to health worker-based interventions that are more expensive and complex to deliver. SMS interventions are seen to be of particular interest in low and middle income countries, where it is difficult to deliver health care into rural and remote communities. SMS also has the advantage of having relatively few side effects ${ }^{4}$. Despite great enthusiasm for using SMS based interventions to improve adherence and improve health outcomes, the evidence is limited ${ }^{5}$. In this summary, we report the results of a recent Cochrane review ${ }^{6}$. While other systematic reviews have addressed the question of using mobile phones for all types of medication adherence, previously no systematic review has only looked at SMS to improve adherence for cardiovascular drugs used in secondary prevention of cardiovascular disease. Furthermore, no previous review has conducted a detailed critical appraisal, including the process (e.g. behavioural science techniques vs simple reminders) of SMS development. To the best of our knowledge this systematic review was the first review to include a questionnaire for authors on how SMS were developed.

\section{Review Methods:}

This review followed the methodology of the Cochrane handbook. As per Cochrane guidelines we examined risk of bias and conducted a summary of findings study using GRADEPro. We only included studies where at least $50 \%$ of participants had established arterial occlusive events that included coronary artery disease, cerebrovascular artery disease, peripheral artery disease, and atherosclerotic aortic disease, and who were prescribed lipid lowering, blood pressure lowering and antiplatelet medication. In addition, all authors were contacted to further understand bias in the creation of the SMS. We requested the following information. Is the SMS intervention a reminder? Did the authors describe the process to construct the content of the text messages? Did they evaluate causes for non-adherence in the target population? Were psychological theories used to develop the messages to target the identified behavioural determinants of non-adherence? Were behaviour-change techniques employed to develop the messages? Were different text messages developed according to participants' characteristics? 
Our primary outcomes included: Adherence to treatment (any definition used in trials), 2. Fatal cardiovascular events, non-fatal cardiovascular events, and Combined CVD event (fatal or non-fatal events). Secondary outcomes included adverse effects, LDL-cholesterol and blood pressure.

\section{Review Findings}

This review found seven small (with a total of 1310 participants) and heterogeneous trials, mostly from high income countries. Six of the trials included patients with coronary heart disease, and one on patients with stroke. Only two of the trials had registered protocols. All studies reported on adherence to medications.

Only three out of the seven trials were conducted in a middle income countries, with one study in China (280 participants), one study in Pakistan (200 individuals), and one study in Malaysia (62 participants). Sample sizes of trials ranged from 34 individuals to 521 individuals with a median of 123 individuals. Follow up time of the studies ranged from 30 days (the largest study) to one year (the smallest study) with a median time of eight weeks. The studies used differ substantially in terms of medication reported, adherence scales, which hamper the possibility of meta-analysis.

Six out of seven trials showed some beneficial effect of mobile phone text messaging for medication adherence (Table 1), but the medications studied, the scales that the authors used, and methods of reporting were so diverse that pooling of the results was considered uninformative. Only one study reported on any of our other primary outcomes, all-cause mortality and found two deaths, both in the control arm. No study reported on associated adverse effects. Only one study reported on LDLcholesterol and found a small reduction. Two studies reported on blood pressure: one found a small reduction, and the other no difference.

Four authors replied to our questionnaire on SMS development. none of the trials used established behavioural techniques to develop the content of SMS to address the modifiable causes of adherence, or that the SMS were tailored to patient characteristics. Two sets of authors reported that the SMS that only reminder SMS were sent out, five study authors reported that an automated system of SMS delivery, and one did not specify, but it is assumed that it did.

\section{Limitations}

All trials had either high or unclear risk of bias for at least two domains, and only one had unclear or high risk of bias for at least four domains. This combined with the high level of heterogeneity, small study size and short duration meant that their results were classed as having very low quality. Moreover, all the studies from middle income countries came from cosmopolitan centres with, which limits the ability to transfer their findings to rural populations and low income countries.

\section{Areas in need of further study:}

This review shows the need for larger trials with longer follow-up and the use of outcomes of higher clinical relevance. Given the dual problem of high burden of atherosclerotic cardiovascular disease and poor adherence to cardiovascular medications, new trials should have a larger impact if conducted in low and middle-income countries. One such ongoing study is the TXT2Heart randomised trial being conducted in Colombia (https://clinicaltrials.gov/ct2/show/NCT03098186) this trial currently has recruited more than 880 participants to date and aims to recruit 1600 individuals and follow them for one year. However even this study is being conducted in urban settings, so to our knowledge no study has been conducted in rural regions. Given the rapid growth of smartphones worldwide, including in low and middle income countries, future studies should also consider the use of personalised health app addressed to tackle adherence 
We recommend that future studies should:

1. Use established behavioural techniques to construct SMS that aimed to tackle modifiable causes of non-adherence

2. Use more relevant outcomes, such as surrogate end-points e.g. LDL-C and blood pressure, or ideally CV events

3. Have extended follows up, of at least one year

4. Have a sample size that allows to detect reasonable changes in clinically relevant end-point

5. Use standardised scales to measure adherence

\section{Conclusions}

While available evidence suggests that SMS are an effective strategy for promoting adherence to medications for secondary prevention of CVD, the available evidence is of low quality from small, short term trials. More research has to be done, particularly in rural regions and low and middle income countries to increase the evidence.

Table 1. Summary of Findings table

Mobile phone text messaging to improve medication adherence in secondary prevention of cardiovascular disease

Patient or population: patients with established arterial occlusive events

Setting: hospital/cardiac rehabilitation facility

Intervention: mobile phone text messaging

Comparison: no intervention or other modes of communication

\begin{tabular}{|c|c|c|c|}
\hline Outcomes & Impact & $\begin{array}{l}\text { ine of participants } \\
\text { (studies) }\end{array}$ & $\begin{array}{l}\text { Quality of the evidence } \\
\text { (GRADE) }\end{array}$ \\
\hline Adherence to treatment & $\begin{array}{l}\text { Six out of seven trials showed } \\
\text { a beneficial effect of mo- } \\
\text { bile phone text messaging for } \\
\text { medication adherence. One } \\
\text { trial showed an improved ad- } \\
\text { herence score for the control } \\
\text { group compared to the inter- } \\
\text { vention group (smallest and } \\
\text { shortest trial) }\end{array}$ & $\begin{array}{l}1310 \\
\text { (7 RCTs) }\end{array}$ & $\begin{array}{l}\oplus O O \bigcirc \\
\text { VERY LOW } 123\end{array}$ \\
\hline
\end{tabular}

GRADE Working Group grades of evidence

High quality: We are very confident that the true effect lies close to that of the estimate of the effect

Moderate quality: We are moderately confident in the effect estimate: The true effect is likely to be close to the estimate of the effect, but there is a possibility that it is substantially different

Low quality: Our confidence in the effect estimate is limited: The true effect may be substantially different from the estimate of the effect

Very low quality: We have very little confidence in the effect estimate: The true effect is likely to be substantially different from the estimate of effect

\footnotetext{
${ }^{1}$ Downgraded one level of evidence due to unclear or high risk of bias for all studies in at least one domain.

${ }^{2}$ Downgraded one level of evidence due to inconsistent reporting of outcome.

${ }^{3}$ Downgraded one level of evidence due to imprecision of the results in one study and different direction of effect in another study.
}

Acknowledgements Norma C Serrano, Javier Mariani, Carlos D Tajer, and Onikepe O Owolabi are authors on the systematic review discussed here, and we thank them for their support. 
The Corresponding Author has the right to grant on behalf of all authors and does grant on behalf of all authors, an exclusive licence (or non exclusive for government employees) on a worldwide basis to the BMJ Publishing Group Ltd and its Licensees to permit this article (if accepted) to be published in HEART editions and any other BMJPGL products to exploit all subsidiary rights.

AJA, NM, and CF all declare that they have no conflicts of interest. Pablo Perel has received a grant from MRC to develop a pilot trial to evaluate efficacy of text message to improve adherence to cardiovascular medications in secondary prevention. JP Casas is the primary supervisor of a PhD student funded through an industrial case EPSRC studentship, where the industrial partner is GSK.

References:

1. Chowdhury R, Khan H, Heydon E, Shroufi A, Fahimi S, Moore C, et al. 2013. Adherence to cardiovascular therapy: a meta-analysis of prevalence and clinical consequences. European Heart Journal 2013;34(38):2940-8.

2. Lozano R, Naghavi M, Foreman KT, Lim S, Shibuya K, Aboyans V, et al. 2012. Global and regional mortality from 235 causes of death for 20 age groups in 1990 and 2010: a systematic analysis for the Global Burden of Disease Study 2010. Lancet 2012;380:2095-128.

3. Sharma P, Agarwal P. Mobile phone text messaging for promoting adherence to antiretroviral therapy in patients with HIV infection. RHL commentary http://apps.who.int/rhl/hivaids/cd009756 sharmap'com/en/ (accessed 4 February 2015).

4. Anglada-Martinez $\mathrm{H}$, Riu-Viladoms $\mathrm{G}$, Martin-Conde $\mathrm{M}$, Rovira-Illamola $\mathrm{M}$, Sotoca-Momblona JM, Codina-Jane C. 2015. Does mHealth increase adherence to medication? Results of a systematic review. International Journal of Clinical Practice 2015;69(1):9-32.

5. Free C, Phillips G, Galli L, Watson L, Felix L, Edwards P, et al. 2013. The effectiveness of mobilehealth technology based health behaviour change or disease management interventions for health care consumers: a systematic review. PLoS Medicine 2013;10(1):e1001362.

6. Adler AJ, Martin N, Mariani J, Tajer CD, Serrano NC, Casas JP, Perel P. Mobile phone text messaging to improve medication adherence in secondary prevention of cardiovascular disease. Cochrane Database Syst Rev. (2017) 2017 Apr 29;4:CD011851. 